\title{
Sarcopenia: A Clinical Review in Spinal Cord Injury Patients
}

\author{
Ali Otom ${ }^{1 *}$, Bilal F Shanti ${ }^{2}$, Ihsan F Shanti ${ }^{3}$ and Maram Bani Mustafa ${ }^{4}$ \\ ${ }^{1}$ Senior Consultant in Spinal Injuries and Musculoskeletal Medicine, CEO of Royal \\ Specialty Center for Spine and Musculoskeletal Disorders, A Fellow of the International \\ Spinal Cord Society (ISCOS), King Hussein Medical Center, Jordan \\ ${ }^{2}$ Pain Specialist, Practicing in the Greater Metropolitan, Phoenix, Arizona, USA \\ ${ }^{3}$ Interventional Pain Consultant, Founder and Medical Director of the American Clinic \\ for Interventional Pain and Spine in Amman, Jordan \\ ${ }^{4}$ Lecturer, Counselor, and Psychologist at the Royal Medical Services in Amman, Jordan \\ *Corresponding Author: Ali Otom, Senior Consultant in Spinal Injuries and \\ Musculoskeletal Medicine, CEO of Royal Specialty Center for Spine and \\ Musculoskeletal Disorders, A Fellow of the International Spinal Cord Society \\ (ISCOS), King Hussein Medical Center, Jordan.
}

Received: April 26, 2021

Published: June 23, 2021

(C) All rights are reserved by Ali Otom., et al.

\begin{abstract}
Sarcopenia is a progressive decrease of muscle function, bulk, and strength that occurs gradually as we age and become frail. This results in a decline in physical stamina that affects normal individuals and those with disabilities. Sarcopenia interferes with functionality, productivity, activities of daily living, and poses risks related to deconditioning and predisposition to physical and psychological consequences. The affected individuals become at increased risk for falls, lack of independence, increased fatty tissue, social isolation, and eventual possible depression. Sarcopenia was considered a natural course of the aging process and was not considered a disease. But, recently, with revised consensus on definition, it is now considered a muscle disease that can be acute or chronic. Emphasis here is made at the difference between aging that is a natural process and frailty that is partially reversible.

This clinical review will encompass the latest updates on this syndrome in spinal cord injury ("SCI") patients as it increases the burden of morbidity and mortality. We recognize that the pathophysiology of sarcopenia is complex and intertwined. This manuscript will briefly review the possible implicated etiologies of sarcopenia without going in dept as our goal is emphasis on sarcopenia in spinal cord patients.
\end{abstract}

Keywords: Sarcopenia; Spinal Cord; Muscle Mass

\section{Definition of sarcopenia}

In general, research related to sarcopenia is scarce and is still in embryonic stages. This is reflected in finding an accurate and 'stable' definition. But we know the term originates from Greek; sarx for flesh, and penia for loss [1]. A major clinical review study on sarcopenia published in 2015 emphasizes on the necessity of combining evaluations of the two elements of sarcopenia: loss of muscle mass and loss of muscle strength [2].
But in 2000, it was estimated that the health care cost of sarcopenia in the United States of America was close to 18 billion dollars. The problem will likely become more complex and costly as the general population is aging [3] and likely will become a geriatric clinical nightmare.

In a study considered to be possibly the first epidemiologic study regarding the prevalence of sarcopenia, published in 1998, 
Baumgartner., et al. theorized that prevalence of sarcopenia increased from $13-24 \%$ in persons under 70 years of age to over $50 \%$ in persons 80 years of age or older. Self-reporting of sarcopenia was directly associated to physical disability in both men and women, independent of ethnicity, morbidity, income, age, obesity, and health behaviors [4].

Like any newly studied clinical entity, the definition of sarcopenia get often revised and updated. Lang., et al. opined that sarcopenia implies the loss of muscle mass, but describes a set of cellular level changes, and subsequent outcome processes. The cellular changes include denervation, mitochondrial variations, inflammatory cascades, and hormonal changes. The outcome processes include diminished muscle strength, decreased function and mobility, fatigue, presence of metabolic disorders, and falls/fractures [5].

Sarcopenia and skeletal muscle and neuromuscular changes in SCI

Natural aging process modifies the skeletal muscles and causes atrophy and disuse atrophy. These changes include decrease in contractile properties, in mass, bulk, composition, and material properties of ligaments, tendons and attachments [5]. The aging muscle morphology changes cause loss of both type I and enhanced loss of type II fibers. This affects the muscle function. In turn, the loss of fast motor units and the simultaneous loss of type II fibers result in loss in muscle power [5]. These changes in muscle strength have been associated to decreases in muscle mass as well as reductions in power per unit area and force per unit area, as non-muscle tissue components take over the lost muscle fiber [6].

Another aspect of the aging skeletal muscle is the infiltration of muscle tissue by adipose deposit in the non-functional and nonused muscle unit, as a result of increased expression of the adipocytic phenotype with aging [7]. Research showed that plausible reasons for this adipose expression include "loss of lipid storage capacity in fat depots, altered fatty acid handling causing lipid accumulation, dysdifferentiation of mesenchymal precursors, such as muscle satellite cells and osteoblast precursors, into a partial adipocyte phenotype" [7].

Since skeletal muscles work in harmony with the motor nerve endings and neurons, abnormalities in the aging nervous system can affect the aging muscles as well. This can affect multiple levels including the higher motor cortex, the spinal cord, peripheral neurons, and the neuromuscular junction [7]. At the level of the cord, there is a significant decrease in the number of alpha motor neurons [7]. Similarly, losses in peripheral nerve fibers and alterations of their myelin sheaths are affected by aging [7] as are changes noted in the neuromuscular junction, with reduced number [8]. In addition, studies have shown that aging causes loss of motor units, adaptive sprouting followed by maladaptive sprouting, and continuing recession of terminals, contributing to the progressive weakness [9].

\section{Sarcopenia and protein homeostasis imbalance in SCI}

Skeletal muscle is maintained by a homeostasis of synthesis of protein from free amino acids and the dissociation of muscle protein into free amino acids. With aging, the imbalance between synthesis of degradation result in muscle loss. Aging causes decreased expression of hormonal factors that promote protein synthesis and increased expression of both endocrine and inflammatory factors that negatively contribute to protein balance by promoting protein degradation [5].

Insulin-Like Growth Factor- 1 (IGF-1) promotes protein synthesis in the skeletal muscle. It binds to receptors on skeletal muscle cell surfaces and ignites a cascade of cell pathways which are "anabolic, anticatabolic, and antiapoptotic" [10]. Aging causes a decline of growth hormone, which results in reduced liver IGF-1 production and reduces skeletal muscle production of IGF-1 [5]. In turn, age-related decline in IGF1 production is linked to age-related reductions in protein synthesis and muscle cell function [5].

Sarcopenia and biological, inflammatory, and hormonal changes in SCI

Studies have shown that the increase in pro-inflammatory cytokines (e.g. interleukin [IL]-6, tumor necrosis factor [TNF]- $\alpha$,) are implicated as potential cause in several age-related biological changes [11]. Accordingly, it was demonstrated there is an inverse relationship of inflammatory status with muscle mass and muscle strength [12]. In particular, TNF- $\alpha$ is found to be a central mediator of cellular inflammatory and apoptosis, and plays a significant role in aging [13].

Research demonstrated a potent hormonal role in the skeletal muscle mass. In particular Growth Hormone and testosterone have a direct effect in muscle decline with aging [14]. Testosterone was 
found to induces muscle fiber hypertrophy by controlling protein synthesis and breakdown in the muscle [14]. GH and IGF-1 were found to be essential for the maintenance of lean body and bone mass [15]. Therefore, in combination, hormonal imbalances and decline do contribute to the formation of sarcopenia.

\section{Sarcopenia: Measurements and assessment}

In 2019, multiple researcher and experts in the field of sarcopenia in Europe, along with the Writing Group for the European Working Group on Sarcopenia in Older People 2 (EWGSOP2), and the Extended Group for EWGSOP2, published a landmark consensus review paper on the definition and diagnosis of sarcopenia [16]. That review paper, that we cannot delve into much detail as we focus on spinal cord injury in this manuscript, has updated many items related to sarcopenia including important items that we will paraphrase as a summary so not to change the contents of the new consensus, as following:

- "The primary indicator is low muscle strength"

- "Sarcopenia is now a disease that can be acute or chronic"

- $\quad$ "Development of algorithm for assessment of severity, case finding, and diagnosis"

- $\quad$ "Specific cut-off points for measures that identify and characterize sarcopenia"

- "Emphasis on early detection and better treatment"

- $\quad$ "Low levels of 3 parameters in sarcopenia: muscle strength, muscle quantity, and physical performance as indicators for severity of sarcopenia"

- "Increase awareness of sarcopenia and its risk".

\section{Sarcopenia in spinal cord injury}

Research supports the fact there is significantly higher incidence and risk for musculoskeletal morbidities in adults with spinal cord injury (SCI) than adults with no SCI (82.4\% Versus 47.5\%) [17].

Verdijk., et al. demonstrated that although both sarcopenia and SCI are associated with loss of skeletal muscle function and mass, muscle fiber atrophy with SCI (Types I and II fibers) and aging (Type II fibers) is accompanied by a muscle fiber type-specific reduction in satellite cell content in humans [18]. It was found that SCI is associated with severe atrophy of both the Types I and II muscle fibers, with a shift toward approximately 90\% Type II fibers [18].
In SCI, the complete absence of voluntary muscle contraction accompanied by the total loss of neurological input is followed by a rapid loss of skeletal muscle mass [19]. In particular, lower extremities muscle atrophy is a major contributing factor to metabolic dysregulation after SCI [20]. Similarly, it was found that the accumulation of adipose tissue within and between the muscle groups of the legs can be up to four times greater in those with SCI [21].

Unfortunately, adipose tissue deposition in skeletal muscle is associated with chronic inflammation, impaired lipid metabolism, protein metabolism, glucose intolerance, and decreased strength and mobility in SCI and in general population [22].

Since bone and muscle share several catabolic pathways that lead to variable degrees of disease in SCI patients [23]. The extent of bone to muscle relationship is so strong that in cases of chronic $\mathrm{SCI}$, the skeleton of the inferior third of the femur and upper third of the tibia may sustain $70 \%$ of decreased mineral density [24].

In one study found that the total muscle mass decreases by about $9.5 \%$ within 6 months, and the muscle mass of the lower limbs is decreased by $15.1 \%$ a year after the SCI [25]. Another study published in 2007, showed that most paraplegics lose motivation while aging and stop performing standing or walking, and thus losing the effects of loading of the lower extremities [26].

In SCI, altered function and atrophy of the skeletal system is a polyfactorial end result. It is the product of disuse atrophy, spasticity, and damage at a microvascular level. This leads to the induction of marked morphological and enzyme changes in SCI [27]. Neuronal demise, disturbed vasoregulation, and muscle denervation contributes to the severity of the atrophy of muscles after SCI. Malabsorption leads to protein deficits and muscle catabolism. Hormonal disturbances of the thyroid, gonads, cortisol, and development of insulin resistance lead to sarcopenia a product of protein deficiency [27].

Protein synthesis and degradation for muscular use are essential for the survival of muscles. With SCI and subsequent atrophy due to inactivity, the degree and length of disuse can be detrimental. During the first initial days of disuse, the muscle complex undergoes a slowing of protein synthesis which retards the body's ability to replenish contractile protein for muscle cells. This leads to muscle protein breakdown [28]. Unfortunately, when proteolysis 
becomes greater than protein synthesis, the muscle mass pays for it and it diminishes with resultant altered body composition [29].

To make matters worse for SCI-related sarcopenia, Duckworth., et al. found that SCI is associated with $50 \%$ susceptibility rate in patients tested for problems with glucose intolerance, insulin resistance and diabetes [30]. In fact another study confirmed prevalence of type 2 diabetes mellitus was 3.5 times higher in individuals with SCI (22\%) compared to controls (6\%). It concluded that 50\% of those with paraplegia and $82 \%$ with tetraplegia were found to be diabetic [31].

\section{Sarcopenia: Therapeutic implications}

Much research has recently focused on the scarce and limited therapeutic management of sarcopenia in SCI. In reviewing the literature, the authors of this manuscript conclude that there is no one particular treatment modality that will fix this complex syndrome. Instead, a multidisciplinary multifaceted approach is recommended.

Therapeutics geared towards muscles

Physiotherapy, exercise, and treadmill

Exercise is shown to improve muscle mass and counteract disuse atrophy in SCI [32]. Exercise also was shown to reduce the expression of Myostatin. This latter plays a limiting role in growth, repair, and regeneration of differentiated adult muscle by inhibiting satellite cell activation [33].

\section{Functional electrical stimulation}

Functional Electrical Stimulation (FES) entails a coordinated voluntary upper body exercise combined with electrical stimulation of the leg large muscle groups to produce a near-full-body exercise. In one study done by Lambach., et al. it was found that regular participation in an FES rowing program provided sufficient power to reduce or reverse expected bone loss at the distal femur and tibia in SCI [34].

\section{Epidural electrical stimulation}

Epidural Electrical Stimulation (EES) is a novel technique that brought a significant hope for motor recovery in SCI [35]. EES consists of implanting a pulse generator that delivers trains of selective stimulation to the lumbosacral spinal cord with timing that coincided with the intended movement [36].
Therapeutics geared towards bone

This therapeutic approach is limited in research outcomes. There are no particular guidelines in this field.

\section{Bisphosphonates}

Research has shown that early administration of bisphosphonates and anti-resorptive agents can reduce SCI-related osteoporosis [37]. But many researchers doubt the efficacy and the safety profile of bisphosphonates in patients with SCI.

\section{Sclerostin antagonists}

Animal studies suggest that sclerostin antagonists might be a possible therapeutic option to prevent bone loss after acute and chronic SCI [38].

\section{Parathyroid hormone}

Parathyroid hormone (PTH) is essential in calcium metabolism. The subcutaneous intermittent administration of PTH has been used for two decades as an anabolic therapeutic agent for osteoporosis treatment [39].

\section{Nutritional interventions}

As is known, amino acids contained in diet loaded with protein is needed for the synthesis of muscle protein. The same applies in sarcopenia. Unfortunately, in reviewing the research data there is a lack of conventional recommendation in SCI.

In one study, addition of whey protein vs. a control diet for 12 weeks along with a 30 min home-based resistance exercise via a physical therapist showed a significant increase in gait speed, grip strength, and time to complete chair stands [40].

In SCI, a study showed that women with paraplegia maintained lower caloric and fat intakes, fewer key nutrients falling outside recommended guidelines, and less overweight or obesity. Tetraplegia patients were found to take more calories and had higher basal metabolic rate (BMI), and the majority of the population was overweight or obese. The study recommended nutritional counseling in SCI patients [41].

In reviewing data, many studies emphasized on improving the mean total energy, the fiber intake, supplementation of vitamins A, B5, B7, B9, D, E, potassium, and calcium that were all deficient in many SCI patients. However, protein and carbohydrate intake and 
vitamins B1, B2, B3, B12, C, K, sodium, phosphorus, copper, and zinc exceeded the USDA recommendation. But the fat intake, vitamin B6, iron, and magnesium were within limits [42].

Like any other clinical complex situation, it is imperative to consider a multidisciplinary nutritional approach for addressing sarcopenia in SCI [23].

\section{Conclusion}

Sarcopenia is more than one entity. It is a syndrome. Addressing one issue at a time in SCI patients may prove counter-intuitive. A better understanding of this syndrome will shed more light on its biochemical aspects. This in turn will broaden our horizons to consider more modern and encompassing therapies, such as regenerative therapies, transcription control it relates to RNA molecules, bone-muscle cross-talk, and tailored exercise therapy.

\section{Bibliography}

1. Rosenberg IH. "Sarcopenia: origins and clinical relevance". Journal of Nutrition 127 (1997): E790-E800.

2. Cesari M and Pahor M. Target population for clinical trials on sarcopenia". The Journal of Nutrition Health and Aging 12.7 (2008): 470-478.

3. Janssen I., et al. "The healthcare costs of sarcopenia in the United States". Journal of the American Geriatrics Society 52.1 (2004): 80-85.

4. Baumgartner RN., et al. "Epidemiology of sarcopenia among the elderly in New Mexico". American Journal of Epidemiology 147.8 (1998): 755-763.

5. Lang T., et al. "Sarcopenia: etiology, clinical consequences, intervention, and assessment". Osteoporosis International 21 (2010): 543-559.

6. Doherty TJ. "Invited review: aging and sarcopenia". Journal of Applied Physiology 95 (2003): 1717-1727.

7. Kirkland JL., et al. "Adipogenesis and aging: does aging make fat go MAD?" Experimental Gerontology 37 (2002): 757-767.

8. Ramirez V and Ulfhake B. "Anatomy of dendrites in motoneurons supplying the intrinsic muscles of the foot sole in the aged cat: evidence for dendritic growth and neo-synaptogenesis". Journal of Comparative Neurology 316 (1992): 1-16.
9. Gordon T., et al. "Adaptive and maladaptive motor axonal sprouting in aging and motoneuron disease". Neurology Research 26 (2004): 174-185.

10. Firth SM and Baxter RC. "Cellular actions of the insulin-like growth factor binding proteins". Endocrine Review 23 (2002): 824-854.

11. Ershler WB and Keller ET. "Age-associated increased interleukin-6 gene expression, late-life diseases, and frailty". Annual Review of Medicine 51 (2000): 245-270.

12. Visser M., et al. "Relationship of interleukin-6 and tumor necrosis factor-alpha with muscle mass and muscle strength in elderly men and women: the Health ABC Study". Journal of Gerontology, Series A: Biological Sciences 57.5 (2002): M326-332.

13. Phillips $\mathrm{T}$ and Leeuwenburgh C. "Muscle fiber-specific apoptosis and TNF-alpha signaling in sarcopenia are attenuated by life-long calorie restriction". FASEB Journal 19 (2005): 668670.

14. Ferrando AA., et al. "Differential anabolic effects of testosterone and amino acid feeding in older men". The Journal of Clinical Endocrinology and Metabolism 88.1 (2003): 358-362.

15. Lissett CA., et al. "Effects of growth hormone on bone and muscle". Growth Hormone and IGF Research 10 (2000): S95-101.

16. J Cruz-Jentoft A., et al. "Sarcopenia: revised European consensus on definition and diagnosis". Age Ageing 48.1 (2019): 1631.

17. Rodriguez G., et al. "Musculoskeletal morbidity following spinal cord injury: A longitudinal cohort study of privatelyinsured beneficiaries". Bone 142 (2021): 115700.

18. Verdijk LB., et al. "Reduced satellite cell numbers with spinal cord injury and aging in humans". Medicine and Science in Sports and Exercise 44.12 (2012): 2322-2330.

19. Castro MJ., et al. "Influence of complete spinal cord injury on skeletal muscle within 6 mo of injury". Journal of Applied Physiology 86 (1999): 350-358.

20. Qin W., et al. "Bone and muscle loss after spinal cord injury: organ interactions". Annals of the New York Academy of Sciences 1211 (2010): 66-84.

21. Gorgey AS and Dudley GA. "Skeletal muscle atrophy and increased intramuscular fat after incomplete spinal cord injury". Spinal Cord 45 (2007): 304-309. 
22. Addison 0., et al. "Intermuscular fat: a review of the consequences and causes". International Journal of Endocrinology 2014 (2014): 309570.

23. Invernizzi M., et al. "Spinal Cord Injury as a Model of BoneMuscle Interactions: Therapeutic Implications From in vitro and in vivo Studies". Frontiers in Endocrinology (Lausanne) 11 (2020): 204.

24. Maïmoun L., et al. "Bone loss in spinal cord-injured patients: from physiopathology to therapy". Spinal Cord 44 (2006): 203210.

25. Wilmet E., et al. "Longitudinal study of the bone mineral content and of soft tissue composition after spinal cord section". Paraplegia 33.11 (1995): 674-677.

26. Dionyssiotis Y., et al. "Bone loss and mechanical properties of tibia in spinal cord injured men". Journal of Musculoskeletal and Neuronal Interactions: JMNI 7.1 (2007): 62-68.

27. Scelsi R. "Skeletal muscle pathology after spinal cord injury". Basic and Applied Myology 11.2 (2001): 75-85.

28. Booth FW., et al. "Molecular events underlying skeletal muscle atrophy and the development of effective countermeasures". International Journal of Sports Medicine 4 (1997): S265-269.

29. Dolbow D R and Gorgey A S. "Effects of Use and Disuse on Nonparalyzed and Paralyzed Skeletal Muscles”. Aging and Disease 7.1 (2016): 68-80.

30. Duckworth WC., et al. "Glucose intolerance in spinal cord injury". Archives of Physical Medicine and Rehabilitation 64.3 (1983): 107-110.

31. Bauman WA and Spungen AM. "Disorders of carbohydrate and lipid metabolism in veterans with paraplegia or quadriplegia: a model of premature aging". Metabolism 43.6 (1994): 749756.

32. Iolascon G., et al. "Pharmacological therapy of sarcopenia: past, present and future". Clinical Cases in Mineral and Bone Metabolism 15 (2018): 407-415.

33. Kim JS., et al. "Impact of resistance loading on myostatin expression and cell cycle regulation in young and older men and women". American Journal of Physiology - Endocrinology and Metabolism 288.6 (2005): E1110-1119.
34. Lambach RL., et al. "Bone changes in the lower limbs from participation in an FES rowing exercise program implemented within two years after traumatic spinal cord injury". Journal of Spinal Cord Medicine 43.3 (2020): 306-314.

35. Cha S., et al. "Spasticity and preservation of skeletal muscle mass in people with spinal cord injury". Spinal Cord 57.4 (2019): 317-323.

36. Wagner FB., et al. "Targeted neurotechnology restores walking in humans with spinal cord injury". Nature 563.7729 (2018): 65-71.

37. Chang KV., et al. "Effectiveness of bisphosphonate analogues and functional electrical stimulation on attenuating post-injury osteoporosis in spinal cord injury patients- a systematic review and meta-analysis". PLoS One 8.11 (2013): e81124.

38. Zhao W., et al. "Sclerostin Antibody Reverses the Severe Sublesional Bone Loss in Rats After Chronic Spinal Cord Injury". Tissue 103.4 (2018): 443-454.

39. Lindsay R., et al. "Teriparatide for osteoporosis: importance of the full course". Osteoporosis International 27.8 (2016): 23952410 .

40. Kang L., et al. "Effects of whey protein nutritional supplement on muscle function among community-dwelling frail older people: A multicenter study in China". Archives of Gerontology and Geriatrics 83 (2019): 7-12.

41. Groah SL., et al. "Nutrient intake and body habitus after spinal cord injury: an analysis by sex and level of injury". Spinal Cord Medicine 32.1 (2009): 25-33.

42. Farkas GJ., et al. "Nutritional status in chronic spinal cord injury: a systematic review and meta-analysis". Spinal Cord 57.1 (2019): 3-17.

\section{Volume 4 Issue 7 July 2021}

(C) All rights are reserved by Ali Otom., et al. 